\title{
Reconstruction of a Tissue Shear Modulus Together with Mechanical Sources
}

\author{
CHIKAYOSHI SUMI*, SAYAKA SUEKANE
}

\author{
Department of Information and Communication Sciences, Faculty of Science and Technology, Sophia University, \\ 7-1 Kioi-cho, Chiyoda-ku, Tokyo 102-8554, Japan
}

\begin{abstract}
To achieve a differential diagnosis of cancerous disease in human soft tissues in vivo in organs such as the liver, breast etc., of cardiac dynamics with myocardial infarction etc. and hemodynamics or atherosclerosis etc., various strain measurement based shear modulus reconstruction methods have been developed. The strain tensor in a region of interest (ROI) is measured using ultrasound (US) or magnetic resonance (MR) imaging. The shear modulus reconstruction methods have also been applied to confirm the effectiveness of thermal treatments such as in human liver in vivo, e.g., regeneration, coagulation etc. In this study, the previously developed shear modulus reconstruction methods are extended, so that arbitrary internal mechanical sources expressed as a static or dynamic pressure, or as a force vector in a region of interest can be reconstructed together with a shear modulus such as a high intensity focused ultrasound (HIFU) and a radiation force for a tissue deformation as well as for treatments, static compressors, vibrators, heart motion and pulsation. Originally, the methods assumed that mechanical sources existed outside of a ROI (i.e., external sources). The new methods are also expected to be able to deal with an internal mechanical source combined with other unknowns, except for the shear modulus, e.g., inertia and mean normal stress. For the purpose of shear modulus reconstructions, such a decrease in the number of unknowns decrease computational time and increase the reconstruction accuracy and stability. The performed simulations show that the extended reconstruction methods have a high potential for yielding an arbitrary internal mechanical source reconstruction together with shear modulus reconstruction. The extension of the reconstruction methods will increase the applications of shear modulus reconstruction, e.g., a deeply situated tissue can be dealt with, and an image during a treatment with a HIFU or radiation force transmission also becomes possible. Moreover, the reconstruction of a mechanical source permits an evaluation of the activities of dynamic or static cells and tissues such as cardiac cells, blood vessels, skin, muscle, cells and tissues in culture etc. and the estimation of the point spread function (PSF) for designing an US beamformer, and for controlling HIFU treatment and tissue deformation (i.e. elasticity imaging) as well as for other applications.
\end{abstract}

Key Words: mechanical source reconstruction, shear modulus reconstruction, high-intensity focus ultrasound (HIFU), ultrasound (US), nuclear magnetic resonance (NMR)

Received 31 August, 2009, Accepted 13 November, 2009. ${ }^{*}$ Corresponding author: Tel, +81-3-3238-3415; Fax, +81-3-3238-3321 ; e-mail,c-sumi@sophia.ac.jp

doi : $10.3191 /$ thermalmed.25.89

(C) 2009 Japanese Society for Thermal Medicine 


\section{Introduction}

To achieve a differential diagnosis of cancerous disease in human soft tissues in vivo in organs such as the liver, breast etc., of cardiac dynamics with myocardial infarction etc. and hemodynamics or atherosclerosis etc., various strain measurement based shear modulus reconstruction methods have been developed by Sumi et al, both one-dimensional ${ }^{1-3)}$ and multidimensional (2D and 3D) ${ }^{3-6)}$. The strain tensor in a region of interest (ROI) is measured using ultrasound (US) ${ }^{7-9)}$ or magnetic resonance (MR) ${ }^{10)}$ imaging. In a dynamic deformation case, inertia or density can both be reconstructed by measuring an acceleration vector. In Sumi, 20053), shear modulus reconstruction as well as strain measurement was applied to confirm the effectiveness of thermal treatments in human liver in vivo, e.g., regeneration, coagulation etc. Shear modulus reconstruction and strain measurement will be used as an effective tool for both diagnosis and monitoring of treatment.

For multidimensional reconstructions, Methods $\mathrm{A}$ to $\mathrm{C}^{4,5)}$ use the mean normal stress as an unknown, whereas Method $\mathrm{F}^{3)}$ uses a typical Poisson ratio (e.g. 0.495). Methods A to C permit dealing with completely incompressible tissues. In addition, in these methods, by using an iterative solution (e.g., a conjugate gradient method) with no references, a shear modulus reconstruction dependent on the initial estimate for the iterative solution can be obtained (Method D), whereas by using a quasi-reference shear modulus (e.g., unity) instead of an absolute shear modulus, a relative shear modulus reconstruction can also be achieved (Method E) ${ }^{6,9)}$. Because no geometrical artifacts are generated, both of the relative reconstructions are useful when the target tissue is deeply situated and it is difficult to set an absolute reference shear modulus (e.g., in the liver and heart). Methods A to F are also summarized both in refs. [6] and [9]. Although all 3D reconstructions can deal with arbitrary mechanical sources that exist outside a region of interest (ROI), i.e., external sources such as a high-intensity focused ultrasound (HIFU), static compressors, vibrators, heart motion and pulsation, if a mechanical source exists inside of a ROI (i.e. an internal source), no shear modulus reconstructions can be achieved.

In this study, the shear modulus reconstruction methods $A$ to $F$ are extended so that arbitrary internal mechanical sources expressed as a static or dynamic pressure or a force vector can be reconstructed together with a shear modulus. These mechanical source reconstructions will increase the applications for a shear modulus reconstruction. Recently, in a research setting, a HIFU or a radiation force ${ }^{10,11)}$ was also used to deform a deeply situated tissue which was inaccessible from the body's surface $^{12,13)}$. Thus, imaging of a treatment and a diagnosis during the HIFU or radiation force transmission will be possible. More accurate shear modulus reconstructions will also be possible for deeply situated tissues (e.g. liver etc.). Moreover, because the reconstruction of a thermal source was also achieved ${ }^{14)}$ by a thermal inverse problem ${ }^{15,16)}$ using simulated US or MR temperature measurements, the thermal reconstruction with a mechanical source reconstruction will permit the evaluation of energy conversion efficiency in tissues from US energy into thermal energy. The reconstructions can also be used as an estimate for the point spread function (PSF) for US for planning and controlling HIFU treatments ${ }^{6,7,14-16)}$ and the radiation force for imaging tissue deformation or shear wave propagation (i.e. tissue elasticity imaging $)^{6,7,10-13)}$, and for designing an US transmission/reception beamformer for US imaging $^{6-8)}$ and for other applications. Because the estimation of the PSF for the HIFU/radiation force can also be performed by using the received echo data, the approach will be an alternative one. In 
addition, the reconstructions using a high frequency US $(\approx 200 \mathrm{MHz})$ will also permit the evaluation of activities of dynamic or static stresses on cells and tissues, e.g. cardiac cells, blood vessels, skin, muscle, cells and tissues in culture etc.

In section "Reconstruction methods", there is a summary of the number of independent fields required to achieve the reconstructions under the conditions in which various types of internal mechanical sources exist. After the feasibility of these reconstructions is verified through simple simulations, conclusions are provided.

\section{Methods}

\section{Reconstruction methods}

With the new reconstruction methods derived from the original Methods A to F, the following motion equations are used respectively for Methods $\mathrm{A}$ to $\mathrm{C}^{4,5)}$ and for the corresponding Methods $\mathrm{D}$ and $\mathrm{E}^{6,9)}$, i.e. simultaneous first order partial differential equations,

$$
\rho \alpha_{\mathrm{i}}=\mathrm{p}_{, \mathrm{j}} \delta_{\mathrm{ij}}+2 \varepsilon_{\mathrm{ij}} \mathrm{G}_{, \mathrm{j}}+2 \varepsilon_{\mathrm{ij}, \mathrm{j}} \mathrm{G}+\mathrm{h}_{\mathrm{i}},
$$

and for Method $\mathrm{F}^{3)}$ and the corresponding Methods $\mathrm{D}$ and $\mathrm{E}^{6,9)}$,

$$
\rho \alpha_{\mathrm{i}}=\left\{\varepsilon_{\alpha \alpha} \delta_{\mathrm{ij}}\right\} \frac{2 \nu}{1-2 \nu} \mathrm{G}_{, \mathrm{j}}+\left\{\varepsilon_{\alpha \alpha} \delta_{\mathrm{ij}}\right\}_{, \mathrm{j}} \frac{2 \nu}{1-2 \nu} \mathrm{G}+2 \varepsilon_{\mathrm{ij}} \mathrm{G}_{\mathrm{j}}+2 \varepsilon_{\mathrm{ij}, \mathrm{j}} \mathrm{G}+\mathrm{h}_{\mathrm{i}},
$$

where $\mathrm{G}$ is the target shear modulus, $\mathrm{h}_{\mathrm{i}}$ is the target mechanical source (expressed here as a force vector), $\mathrm{p}$ is the mean normal stress, $\nu$ is the Poisson ratio, $\varepsilon_{\mathrm{ij}}$ is the strain tensor, and $\alpha_{\mathrm{i}}$ is the acceleration vector. We use the rectangular Cartesian frame of the reference with $\mathrm{x}_{1}-, \mathrm{x}_{2}-$, and $\mathrm{x}_{3}$-axes. An indicial notation is used to take advantage of the summation convention in which the repetition of an index in a term denotes the summation with respect to the index over its range. We also follow a commonly used practice in which a comma in an index denotes partial differentiation such that ",j" denotes a partial derivative with respect to the $x_{j}$-axis. Here, the mechanical source term $h_{i}$ which is set to zero throughout a ROI in the original shear modulus reconstruction methods $\mathrm{A}$ to $\mathrm{F}^{6,9)}$ is considered together.

Basically, dynamic deformation cases are to be dealt with. That is, the inertia term $\rho \alpha_{\mathrm{i}}$ is also used to determine the inertia term $\rho \alpha_{\mathrm{i}}$ or the density $\rho$ together with the shear modulus $\mathrm{G}$ and the mechanical source $h_{i}$ or the mean normal stress $\mathrm{p}$, etc. When static deformation cases are examined, equilibrium equations are used instead (i.e., $\rho \alpha_{\mathrm{i}}=0$ ). In these equations, the measured strain tensor $\varepsilon_{\mathrm{ij}}$ or the acceleration vector $\alpha_{\mathrm{i}}$ is used with spatially inhomogeneous coefficients. References (i.e. the initial conditions) used in Methods A to E using the discretization methods such as the finite difference method (FDM) or the finite element method (FEM) are summarized both in refs. [6] and [9]. For both deformation cases, the references to be used, the unknowns and the number of unknowns are also summarized in Table I in this report. That is, for Method A, the reference shear modulus; for Method $\mathrm{B}$, references of mean normal stress and shear modulus; for Method C, quasi-reference mean normal stress and reference shear modulus; for Method F, the reference shear modulus and typical Poisson ratio over the ROI ; for Method D, there is no use of the references nor quasi-references in Methods A to C and F ; for Method E, a quasi-reference shear modulus is used instead of the reference shear modulus in Methods A to $\mathrm{C}$ and F. As shown in Table I, instead of the reference and quasi-reference shear moduli, those of 
Table I. For an external mechanical source ${ }^{6,9)}$, the setting of references and the number of unknowns for Methods A-E of the shear modulus $G$ and the mean normal stress p. For a static deformation case, the use of reference density $\rho$ is removed, i.e., no unknown variables with density $\rho$ nor inertia $\rho(\rho \alpha)$. Quasi and relative reconstructions to be obtained are shown using a bar. U, reference is used ; -, reference is not used ; Q, quasi-reference is used. Bracket in the case where the use in finite element method is different from that in finite difference method. All methods require a measured deformation field.

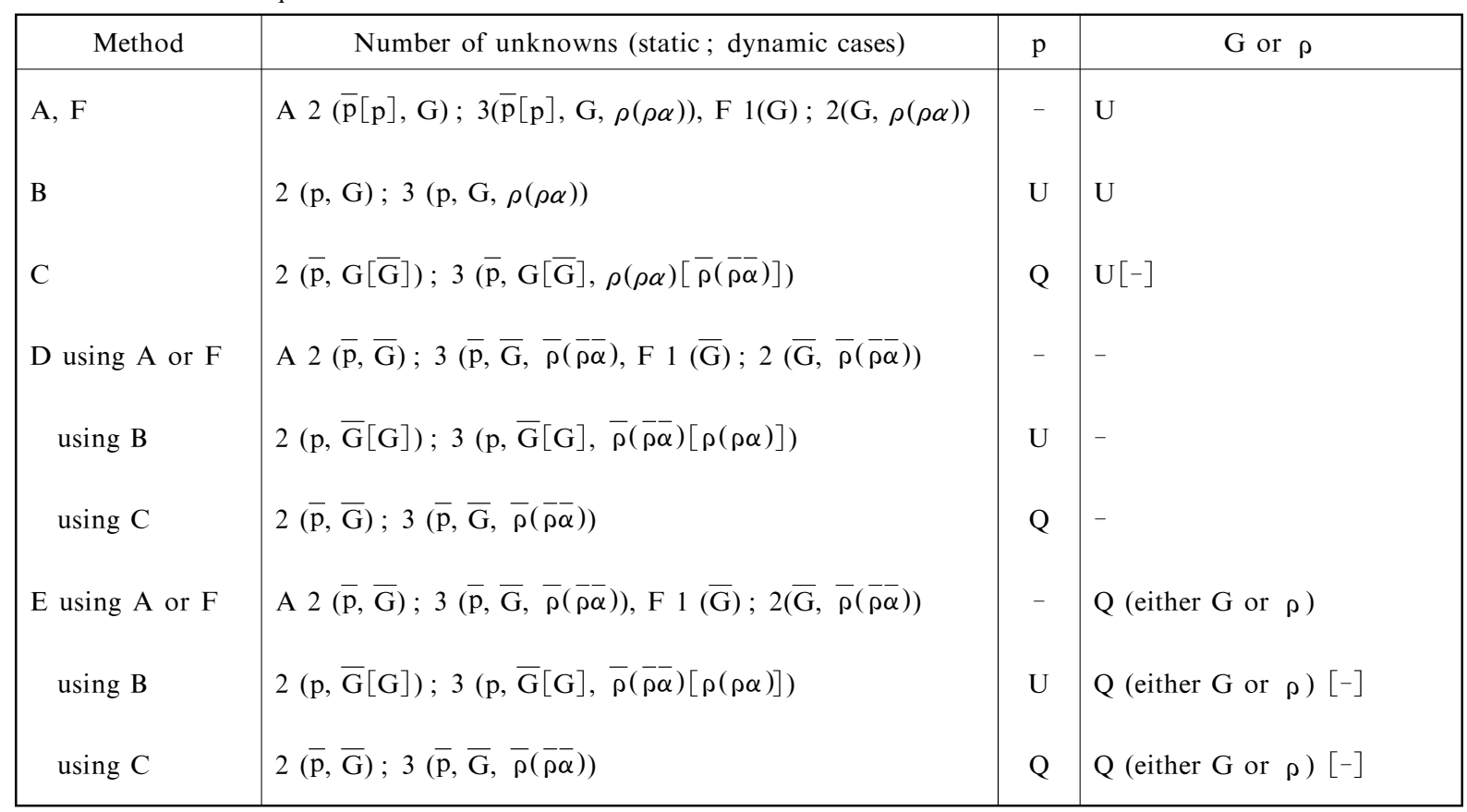

densities can also be used properly. All these reconstruction methods require a measured deformation field. When the target tissue is compressible, by using Method B, the Poisson ratio $\nu$ can also be reconstructed from the reconstructed mean normal stress $\mathrm{p}$ and the measured volume strain $\varepsilon_{\alpha \alpha}{ }^{4,55}$.

As mechanical source models, the static or dynamic (variable) pressure p' $\left(=h_{1}=h_{2}=h_{3}\right)$, or the force vector hi' can be considered. However, for instance with 3D reconstructions, the number of unknowns in the equations cannot be larger than three. In this inverse problem, to yield a unique and stable approximation to each reconstruction target, the number of equations should be larger than that of unknowns at least. Thus, if necessary, a typical density will be used, or more equations will be used by measuring independent plural deformation fields, or it will be assumed that the mechanical source is stationary, and be assumed that the same mechanical source is present in some dimensions. For instance, for a dynamic deformation case using Method C, by using two independent deformation fields measured at two different times, the unknowns describing mechanical pressures and mean normal stresses at the two different times can be determined together with the shear modulus and density (i.e. six unknowns). Moreover, for a 3D force vector hi', similarly in a static deformation case, only the quasi-mechanical source $\mathrm{H}^{\prime}$ can be determined under the assumption that $\mathrm{H}^{\prime}=\mathrm{h} 1^{\prime}=h 2^{\prime}=h 3^{\prime}$, although the pressure $\mathrm{p}^{\prime}$ can be determined.

For all methods except Method $\mathrm{E}$ that deals with the logarithm of the shear modulus as an 
Table II. For an internal mechanical source, in a static deformation case $(\rho \alpha=0)$, the number of independent fields required by Methods F, A to C, D, E, A', D' and E' with the number of unknowns (no prime number). The numbers required for reconstructing the internal mechanical sources that exist only in one deformation field using another deformation field together are also shown by prime numbers. $\overline{\mathrm{G}}$ is a quasi-reference of shear modulus.

\begin{tabular}{|c|c|c|c|c|c|c|}
\hline Method (and ref.) & \multicolumn{2}{|c|}{$\begin{array}{l}F(G), D \text { and } E(\bar{G}) \\
\text { using } F\end{array}$} & \multicolumn{2}{|c|}{$\begin{array}{l}\text { A to } C(G), D \text { and } E(\bar{G}) \\
\text { using } A \text { to } C\end{array}$} & \multicolumn{2}{|c|}{$\begin{array}{l}A^{\prime}(G), D^{\prime} \text { and } E^{\prime}(\bar{G}) \\
\text { using } A^{\prime}\end{array}$} \\
\hline Number & Field & Unknown & Field & Unknown & Field & Unknown \\
\hline Pressure p' & 1 & $2\left(\mathrm{G}, \mathrm{p}^{\prime}\right)$ & 1 & $3(\mathrm{G}, \mathrm{p}, \mathrm{p})$ & 1 & $2(\mathrm{G}, \mathrm{comb})$ \\
\hline $\begin{array}{l}\text { 3D force } \\
\text { hi' }(i=1-3)\end{array}$ & $\begin{array}{l}1 \\
2\end{array}$ & $\begin{array}{l}2\left(\mathrm{G}, \mathrm{H}^{\prime}\right) \\
4(\mathrm{G}, \mathrm{hi})\end{array}$ & $\begin{array}{l}1 \\
2\end{array}$ & $\begin{array}{l}3\left(\mathrm{G}, \mathrm{H}^{\prime}, \mathrm{p}\right) \\
6(\mathrm{G}, \mathrm{hi}, \mathrm{p} \times 2)\end{array}$ & $\begin{array}{l}1 \\
1 \\
2\end{array}$ & $\begin{array}{l}2(\mathrm{G}, \mathrm{COMB}) \\
3(\mathrm{G}, \mathrm{COMB}, \mathrm{p}) \\
5(\mathrm{G}, \mathrm{comb} \times 3, \mathrm{p})\end{array}$ \\
\hline $\begin{array}{l}\text { 2D force } \\
\text { hi' }(i=1,2)\end{array}$ & 1 & 3 (G, hi') & $\begin{array}{l}1 \\
2\end{array}$ & $\begin{array}{l}3\left(\mathrm{G}, \mathrm{H}^{\prime}, \mathrm{p}\right) \\
5(\mathrm{G}, \mathrm{hi}, \mathrm{p} \times 2)\end{array}$ & $\begin{array}{l}1 \\
1 \\
2\end{array}$ & $\begin{array}{l}2(\mathrm{G}, \mathrm{COMB}) \\
3(\mathrm{G}, \mathrm{COMB}, \mathrm{p}) \\
4(\mathrm{G}, \mathrm{comb} \times 2, \mathrm{p})\end{array}$ \\
\hline A force h' & 1 & 2 (G, h') & 1 & $3(\mathrm{G}, \mathrm{h}, \mathrm{p})$ & 1 & $2(\mathrm{G}, \mathrm{comb})$ \\
\hline
\end{tabular}

In the table, $\mathrm{G}$ is the shear modulus, $\mathrm{p}$ is the mean normal stress, and comb is obtained by combining terms in Methods $\mathrm{A}^{\prime}$, and D' and E' using A' which can be found by seeing the unknowns in A, and D and E using A. H' and COMB express inaccurate values of hi' and comb, respectively, owing to theoretical limitations. Both of the terms H' and COMB are obtained by the fact that even if the number of independent fields increases, hi' and comb cannot be reconstructed theoretically owing to fewer equations. When using Methods D and E, quasi-reconstructions of mechanical sources are obtained, although not described in table.

unknown ${ }^{6,9,3-5)}$, the mechanical source can be considered together with a mean normal stress or inertia by combining them as unknown terms. For instance, for a static deformation case using Method A, when a mechanical source is combined with a mean normal stress, the method is referred to as Method A'. The obtained combined term is referred to as a "comb". Similarly, in a dynamic deformation case, for Method A', by combining the mechanical source with a mean normal stress, the unknowns are the shear modulus, the density or the inertia, and the combined term "comb" (i.e. three unknowns), whereas for Method A", by combining a mechanical source with inertia, the unknowns are the shear modulus, the mean normal stress, and the combined term "comb" (i.e. three unknowns). Moreover, for Method A"”, by combining the mechanical source, inertia and mean normal stress, the unknowns are only the shear modulus and the combined term "comb" (i.e. two unknowns). Thus, except for Method A, Methods A', $\mathrm{A}$ " and A" require only one deformation field (i.e. three equations).

In a manner similar to that with Methods A', A" and A" with respect to Method A, by combining unknowns, Methods F", B" and C", D' and E' using A' or F', D" and E" using A" to C" and F", and D" and E" using A" can be obtained from the corresponding Methods F, B, C, D and E. For these methods, the number of independent fields required to achieve shear modulus reconstructions under conditions in which an internal mechanical source exists are summarized. For static mechanical sources, the numbers required are shown in Table II, whereas for dynamic mechanical sources, those required are shown by no prime numbers (one or two) in Table III. In the tables, the reference or quasi-reference (bar) shear modulus or density to be used is also shown. $G$ is the shear modulus, $p$ is the mean normal stress, and comb term is obtained by combining terms which can be found by determining the unknowns 
Table III. For an internal mechanical source, in a dynamic deformation case, the number of independent fields required by Methods F, F", A to C, A', A" to C", A"', D and E, D' and E', D" and E”, and D"” and E" with the number of unknowns (no prime number). The numbers required for reconstructing the internal mechanical sources that exist only in one deformation field using another deformation field together are also shown by prime numbers.

\begin{tabular}{|c|c|c|c|c|c|c|}
\hline Method (and ref.) & \multicolumn{2}{|c|}{$\begin{array}{l}F(G), D \text { and } E(\bar{G} \text { or } \bar{\rho}) \\
\text { using } F\end{array}$} & \multicolumn{2}{|c|}{$\begin{array}{l}\text { F" }(G), D ” \text { and } E "(\bar{G} \text { or } \bar{\rho}) \\
\text { using F" }\end{array}$} & \multicolumn{2}{|c|}{$\begin{array}{l}\text { A to } C(G \text { or } \rho), D \text { and } E(\bar{G} \text { or } \\
\bar{\rho}) \text { using } A \text { to } C\end{array}$} \\
\hline Number & Fields & Unknown & Fields & Unknown & Fields & Unknown \\
\hline Pressure p' & 1 & $3\left(\mathrm{G}, \mathrm{p}^{\prime}, \rho\right)$ & 1 & $2(\mathrm{G}, \mathrm{comb})$ & $\begin{array}{l}2 \\
2\end{array}$ & $\begin{array}{l}6\left(\mathrm{G}, \mathrm{p}^{\prime} \times 2, \rho, \mathrm{p} \times 2\right) \\
5\left(\mathrm{G}, \mathrm{p}^{\prime}, \rho, \mathrm{p} \times 2\right)\end{array}$ \\
\hline $\begin{array}{l}\text { 3D force } \\
\text { hi' }(i=1-3)\end{array}$ & $\begin{array}{l}1 \\
2,\end{array}$ & $\begin{array}{l}3\left(\mathrm{G}, \mathrm{H}^{\prime}, \rho\right) \\
5(\mathrm{G}, \mathrm{hi}, \rho)\end{array}$ & $\begin{array}{l}1 \\
2\end{array}$ & $\begin{array}{l}2(\mathrm{G}, \mathrm{COMB}) \\
4(\mathrm{G}, \mathrm{comb} \times 3)\end{array}$ & $\begin{array}{l}2 \\
2\end{array}$ & $\begin{array}{l}6\left(\mathrm{G}, \mathrm{H}^{\prime} \times 2, \rho, \mathrm{p} \times 2\right) \\
5\left(\mathrm{G}, \mathrm{H}^{\prime}, \rho, \mathrm{p} \times 2\right)\end{array}$ \\
\hline $\begin{array}{l}\text { 2D force } \\
\text { hi' }(i=1,2)\end{array}$ & $\begin{array}{l}2 \\
1 \\
2\end{array}$ & $\begin{array}{l}6\left(\mathrm{G}, \text { hi' }^{\prime} \times 2, \rho\right) \\
3\left(\mathrm{G}, \mathrm{H}^{\prime}, \rho\right) \\
4\left(\mathrm{G}, \mathrm{hi}^{\prime}, \rho\right)\end{array}$ & $\begin{array}{l}2 \\
1 \\
1\end{array}$ & $\begin{array}{l}5(\mathrm{G}, \mathrm{comb} \times 4) \\
3(\mathrm{G}, \mathrm{comb} \times 2) \\
2(\mathrm{G}, \mathrm{COMB})\end{array}$ & $\begin{array}{l}2 \\
2 \\
2\end{array}$ & $\begin{array}{l}6\left(\mathrm{G}, \mathrm{H}^{\prime} \times 2, \rho, \mathrm{p} \times 2\right) \\
5\left(\mathrm{G}, \mathrm{H}^{\prime}, \rho, \mathrm{p} \times 2\right) \\
6(\mathrm{G}, \mathrm{hi}, \rho, \mathrm{p} \times 2)\end{array}$ \\
\hline A force $h$ ' & 1 & $3(\mathrm{G}, \mathrm{h}, \mathrm{p})$ & 1 & $2(\mathrm{G}, \mathrm{comb})$ & $\begin{array}{l}2 \\
2\end{array}$ & $\begin{array}{l}6\left(\mathrm{G}, \mathrm{h}^{\prime} \times 2, \rho, \mathrm{p} \times 2\right) \\
5(\mathrm{G}, \mathrm{h}, \rho, \mathrm{p} \times 2)\end{array}$ \\
\hline
\end{tabular}

\begin{tabular}{|c|c|c|c|c|c|c|}
\hline Method (and ref.) & \multicolumn{2}{|c|}{$\begin{array}{l}A^{\prime}(G \text { or } \rho), D^{\prime} \text { and } E^{\prime}(\bar{G} \text { or } \\
\bar{\rho}) \text { using } A^{\prime}\end{array}$} & \multicolumn{2}{|c|}{$\begin{array}{l}A " \text { to } C "(G), D " \text { and } E "(\bar{G}) \\
\text { using } A " \text { to } C "\end{array}$} & \multicolumn{2}{|c|}{$\begin{array}{l}A ",(G), D ", \text { and E"” } \\
(\bar{G}) \text { using A"” }\end{array}$} \\
\hline Number & Fields & Unknown & Fields & Unknown & Fields & Unknown \\
\hline Pressure p' & $\begin{array}{l}1 \\
2\end{array}$ & $\begin{array}{l}3(\mathrm{G}, \text { comb }, \rho) \\
4(\mathrm{G}, \mathrm{comb}, \rho, p)\end{array}$ & $\begin{array}{l}1 \\
2\end{array}$ & $\begin{array}{l}3(\mathrm{G}, \text { comb, } \mathrm{p}) \\
4(\mathrm{G}, \mathrm{comb}, \mathrm{p} \times 2)\end{array}$ & 1 & $2(\mathrm{G}, \mathrm{comb})$ \\
\hline $\begin{array}{l}\text { 3D force } \\
\text { hi' }(i=1-3)\end{array}$ & $\begin{array}{l}1 \\
2 \\
2\end{array}$ & $\begin{array}{l}3(\mathrm{G}, \mathrm{COMB}, \rho) \\
4(\mathrm{G}, \rho, \mathrm{COMB}, \mathrm{p}) \\
6(\mathrm{G}, \rho, \mathrm{comb} \times 3, \mathrm{p})\end{array}$ & $\begin{array}{l}1 \\
2 \\
2\end{array}$ & $\begin{array}{l}3(\mathrm{G}, \mathrm{COMB}, \mathrm{p}) \\
4(\mathrm{G}, \mathrm{COMB}, \mathrm{p} \times 2) \\
6(\mathrm{G}, \mathrm{comb} \times 3, \mathrm{p} \times 2)\end{array}$ & 1 & $2(\mathrm{G}, \mathrm{COMB})$ \\
\hline $\begin{array}{l}\text { 2D force } \\
\text { hi' }(i=1,2)\end{array}$ & $\begin{array}{l}1 \\
2 \\
2\end{array}$ & $\begin{array}{l}3(\mathrm{G}, \mathrm{COMB}, \rho) \\
4(\mathrm{G}, \mathrm{COMB}, \rho, \mathrm{p}) \\
5(\mathrm{G}, \mathrm{comb} \times 2, \rho, \mathrm{p})\end{array}$ & $\begin{array}{l}1 \\
2\end{array}$ & $\begin{array}{l}3(\mathrm{G}, \mathrm{COMB}, \mathrm{p}) \\
5(\mathrm{G}, \mathrm{comb} \times 2, \mathrm{p} \times 2)\end{array}$ & 1 & $2(\mathrm{G}, \mathrm{COMB})$ \\
\hline A force h' & $\begin{array}{l}1 \\
2\end{array}$ & $\begin{array}{l}3(\mathrm{G}, \text { comb, } \rho) \\
4(\mathrm{G}, \mathrm{comb}, \rho, p)\end{array}$ & $\begin{array}{l}1 \\
2\end{array}$ & $\begin{array}{l}3(\mathrm{G}, \mathrm{comb}, \mathrm{p}) \\
4(\mathrm{G}, \mathrm{comb}, \mathrm{p} \times 2)\end{array}$ & 1 & $2(\mathrm{G}, \mathrm{comb})$ \\
\hline
\end{tabular}

In the table, $\mathrm{G}$ is the shear modulus, $\rho$ is the density, $\mathrm{p}$ is the mean normal stress, and comb is obtained by combining terms in Methods F", A', A" to C", A", D' and E' using A', D" and E" using A" to C" and F", D" and E" using A" which can be found by determining the unknowns in F, and A to C, respectively. Similarly in a static case (Table II), H' and COMB express inaccurate values of hi' and comb, respectively. When using Methods D and E, quasi-reconstructions of mechanical sources are obtained (not shown in table).

in the original Methods F, A to C, D and E. H' and COMB express the inaccurate reconstruction of the target hi' and comb, respectively, owing to theoretical limitations. That is, H' and COMB are obtained when hi' and comb cannot be reconstructed, even if the number of independent fields increases. Also note that, Methods $\mathrm{D}$ and $\mathrm{E}$ yields quasi-reconstruction of mechanical sources, i.e., with inaccurate values, although not specifically shown in the tables. In the tables, the numbers required for reconstructing the internal mechanical sources that exist only in one deformation field using another 
deformation field together are also shown by prime numbers. For all the Methods, the set of equations is solved by using a least squares method. If the number of equations that can hold is larger than that of unknowns, the extra equations may be removed from the set of equations.

Although not dealt with, if the dynamic mechanical source is sinusoidal and the frequency and phase can be obtained, by dealing with the magnitude of the mechanical source as an unknown, the dynamic reconstruction problem can be dealt with similarly to the static mechanical source reconstruction problem (i.e., Table II). If the magnitude can be obtained, the frequency will become an unknown.

\section{Preparation of simulations}

A tissue phantom (a cube with $50 \mathrm{~mm}$ sides) and mechanical sources were simulated using a successive-over-relaxation method (see Fig. 1). For instance, a spherical source (10 $\mathrm{mm}$ in diameter) that partially overlapped a central spherical inclusion (10 $\mathrm{mm}$ dia.) having a different shear modulus from that of the surrounding region (e.g., $2 \mathrm{vs} 1 \times 10^{5} \mathrm{~N} / \mathrm{m}^{2}$ ) was used. Specifically, there was an overlap of $5 \mathrm{~mm}$. For internal mechanical sources, static and dynamic (sinusoidal and step) models were used. Specifically, for the three models, the pressure p' and the 3D force vector hi' were examined (see Table IV, i.e. the mechanical sources (i) to (vi)). For the reconstructions, no prime numbers of deformation fields are used (Tables II and III). In addition to such a small internal mechanical source, in order to obtain a large ROI (a cube $30 \mathrm{~mm}$ on each side), an external large mechanical source was also modeled. That is, the upper surface of the phantom was uniformly compressed in the axial direction. Here, with Methods F, A', A"', C and C", the reference region for the shear modulus was set at the upper surface of a ROI, and a quasi-reference point for mean normal stress (set to zero) was set at the central point of the upper surface. All the results of 3D reconstructions are shown only for the vertical central 2D plane in the ROI, i.e., $\mathrm{y}=25 \mathrm{~mm}$ (see Fig. 1).

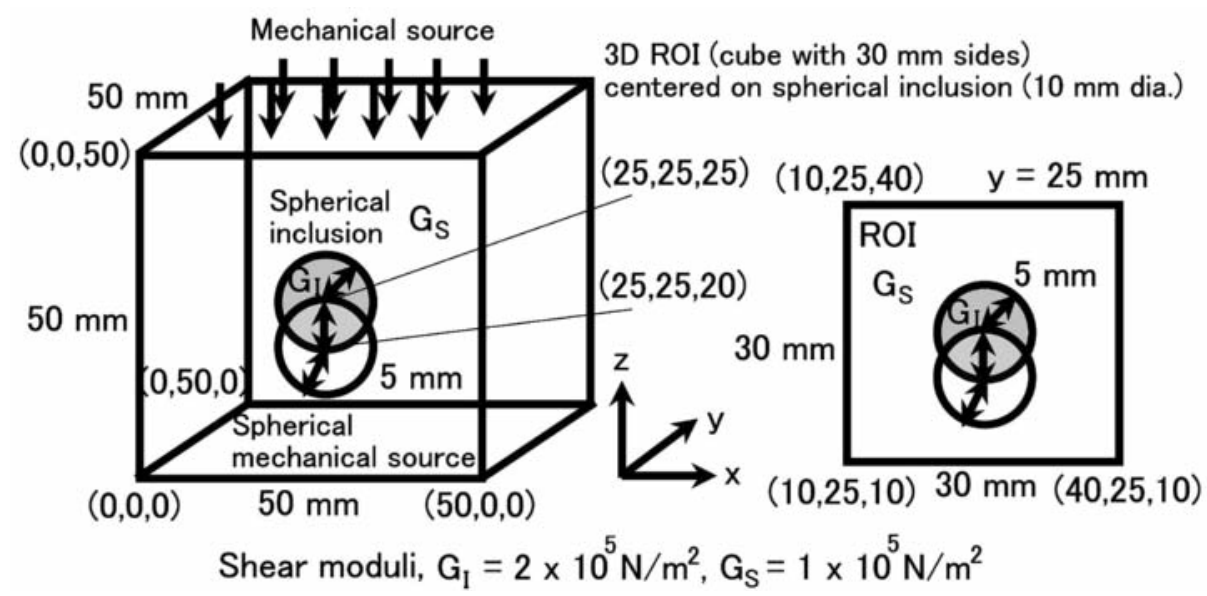

Fig. 1. Simulated cubic phantom ( $50 \mathrm{~mm}$ sides) and vertical central $2 \mathrm{D}$ square ROI with $\mathrm{y}=25 \mathrm{~mm}$ in cubic ROI (30 mm sides) centered on spherical inclusion $(10 \mathrm{~mm}$ dia.). 
Table IV. Means of shear moduli $\left(\times 10^{5} \mathrm{~N} / \mathrm{m}^{2}\right.$, upper $)$ and mechanical sources $\left(\times 10^{4} \mathrm{~N} / \mathrm{m}^{2}\right.$, lower $)$ reconstructed in a $5 \mathrm{~mm}^{2}$ square region in the inclusion using Methods F, C, A' C" and A'”. Standard deviations (SDs) are shown in parentheses.

\begin{tabular}{|c|c|c|c|c|c|}
\hline Method & $\mathrm{F}$ & $\mathrm{C}$ & $\mathrm{A}^{\prime}$ & C" & A"” \\
\hline $\begin{array}{l}\text { (ii) Static } \\
\text { force vector hi' }\end{array}$ & $\begin{array}{l}1.96(0.06) \\
1\end{array}$ & $\begin{array}{l}2.10(0.12) \\
{[9.15(1.32)]^{2}}\end{array}$ & $1.51(0.10)$ & - & - \\
\hline $\begin{array}{l}\text { (iv) Step } \\
\text { pressure p' }\end{array}$ & - & $\begin{array}{l}1.81(0.07) \\
5.28\left(1.53 \times 10^{-11}\right)\end{array}$ & $2.05(0.09)$ & $2.05(0.06)$ & $1.93(0.05)$ \\
\hline $\begin{array}{l}\text { (v) Sinusoidal } \\
\text { force vector hi' }\end{array}$ & - & $2.06(0.07)$ & - & - & $2.05(0.06)$ \\
\hline
\end{tabular}

In the table, - indicates not evaluated ; 1 indicates that although the conjugate gradient method converged, because the result was unstable, the statistics were not evaluated ; 2 indicates that although no accurate result can be obtained theoretically, the statistics were evaluated in a trial.

\section{Results}

\section{Static deformation case}

In a static deformation case, the unknowns are a mechanical source together with a shear modulus for Method $\mathrm{F}$, and a mean normal stress with a shear modulus for Methods $\mathrm{C}$ and $\mathrm{A}$ '. For internal mechanical sources, (i) a pressure p' of $5 \times 10^{4} \mathrm{~N} / \mathrm{m}^{2}$ and (ii) a 3D force vector hi' with $(5,10,15) \times 10^{4}$ $\mathrm{N} / \mathrm{m}^{2}$ were examined.

With Method F, for (i) and (ii), a shear modulus was successfully reconstructed with a high accuracy, i.e. Fig. 2a [(i)] and Fig. 3a [(ii)]. In Table IV the means and standard deviations (SDs) of the shear moduli reconstructed in a $5 \mathrm{~mm}^{2}$ square region in the inclusion are $1.98(0.07)$ and $1.96(0.06) \times 10^{5} \mathrm{~N} /$ $\mathrm{m}^{2}$. However, the mechanical sources could not be accurately reconstructed (they are omitted).

However, when using Method C, for (i) and (ii), the shear modulus and mean normal stresses were reconstructed with high accuracies (mean shear moduli, 1.95 and $2.10 \times 10^{5} \mathrm{~N} / \mathrm{m}^{2}$, see Table IV). In addition, although the static pressure p' $[(\mathrm{i})]$ was accurately reconstructed $\left(4.25 \times 10^{4} \mathrm{~N} / \mathrm{m}^{2}\right)$, with the mechanical sources for the $3 \mathrm{D}$ force vector hi' [(ii)], not the respective components of the $3 \mathrm{D}$ force vector, but a quasi-mechanical source under the assumption of $\mathrm{h}^{\prime}{ }^{\prime}=\mathrm{h} 2^{\prime}=\mathrm{h} 3^{\prime}\left(\equiv \mathrm{H}^{\prime}\right)$ was evaluated owing to the theoretical limitations discussed in the previous section (see the means in Table IV, 9.15 vs the original, $\left.(5,10,15) \times 10^{4} \mathrm{~N} / \mathrm{m}^{2}\right)$. Also see Fig. $2 \mathrm{~b}[(\mathrm{i})]$ and Fig. $3 \mathrm{~b}[(\mathrm{ii})]$.

In addition, meaningfully, for (i), Method $\mathrm{A}^{\prime}$ also yielded accurate shear modulus reconstructions (mean, $2.05 ; \mathrm{SD}, 0.06 \times 10^{5} \mathrm{~N} / \mathrm{m}^{2}$ ). See Fig. 2c [(i)] and Fig. 3c [(ii)]. Method A' was also confirmed to be an effective method. Because there are only two unknowns, the computational time is significantly less than that for Method $\mathrm{C}$ with three unknowns. 
(a)

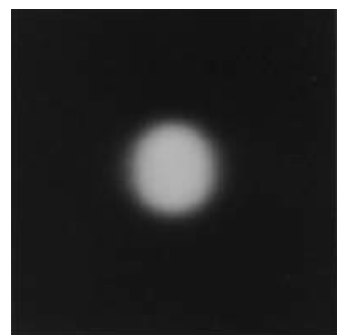

(b)
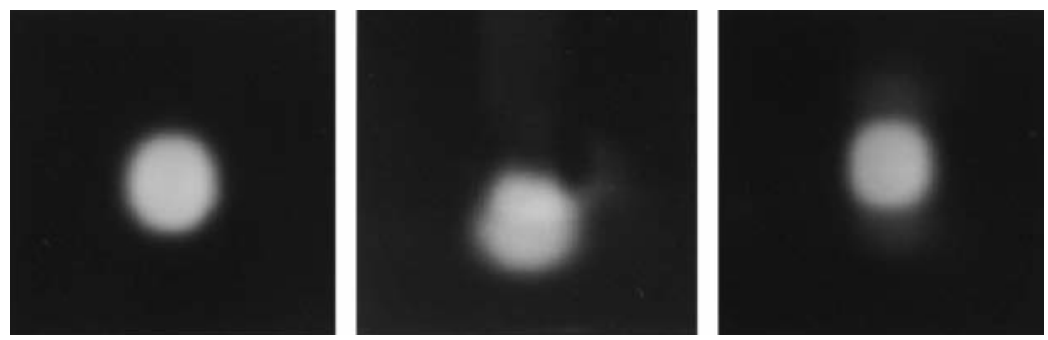

(c)
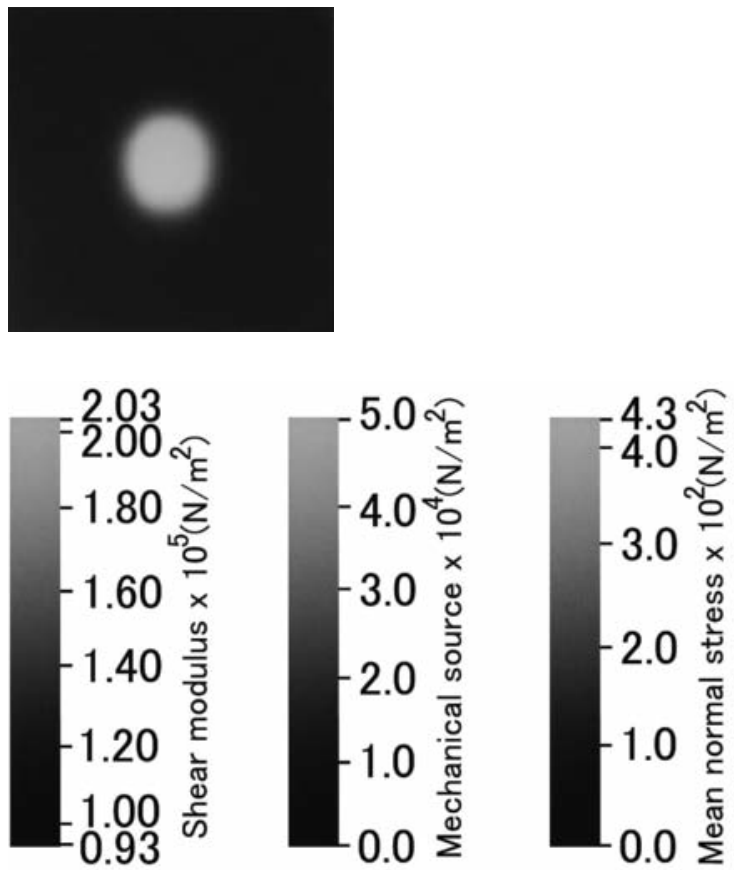

Fig. 2. Reconstructions for an internal mechanical source (vertical central plane, $y=25 \mathrm{~mm}$ ) : (i) static pressure p' using Methods (a) F (shear modulus), (b) $\mathrm{C}$ (shear modulus, mechanical source, mean normal stress), and (c) A' (shear modulus).

\section{Dynamic deformation case}

Similarly, dynamic cases were also dealt with. In these simulations, four internal dynamic mechanical sources were considered, i.e. (iii) a sinusoidal pressure $\left(1 \mathrm{~Hz}, 5 \times 10^{4} \mathrm{~N} / \mathrm{m}^{2}\right)$, (iv) a step mechanical pressure $\left(5 \times 10^{4} \mathrm{~N} / \mathrm{m}^{2}\right),(\mathrm{v})$ a sinusoidal $3 \mathrm{D}$ force vector $\left(1 \mathrm{~Hz},(5,10,15) \times 10^{4} \mathrm{~N} / \mathrm{m}^{2}\right)$ and (vi) a step $3 \mathrm{D}$ force vector $\left[(5,10,15) \times 10^{4} \mathrm{~N} / \mathrm{m}^{2}\right]$. For the sinusoidal and step mechanical sources, the data obtained at 0.25 and $0.50 \mathrm{sec}$ immediately after applying the mechanical source were used. 
(a)

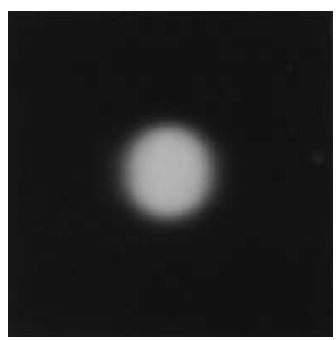

(b)
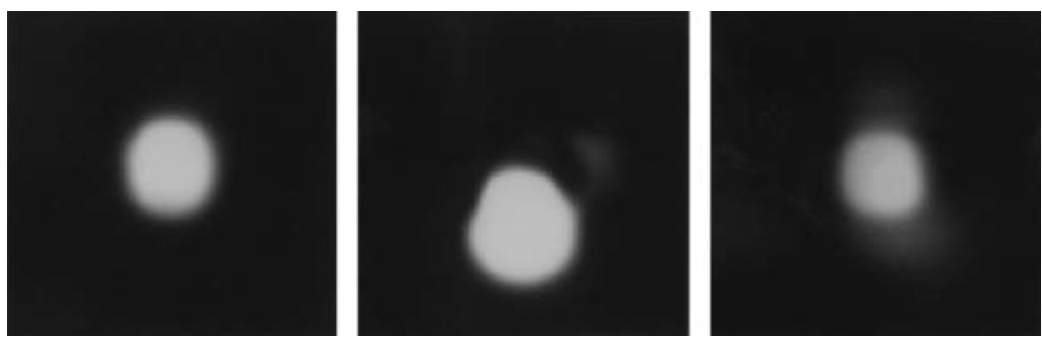

(c)
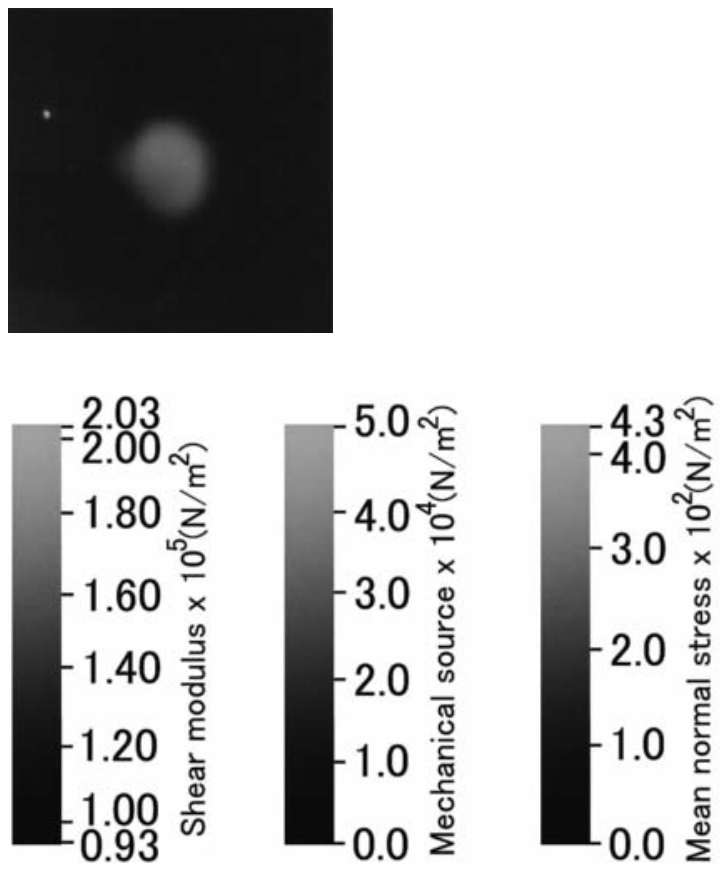

Fig. 3. Reconstructions for an internal mechanical source (vertical central plane, $\mathrm{y}=25 \mathrm{~mm}$ ) : (ii) static 3D force vector hi' using Methods (a) F (shear modulus), (b) C (shear modulus, mechanical source, mean normal stress), and (c) A' (shear modulus).

Figure 4 shows the shear modulus reconstructions obtained for (iii) using Methods (a) C, (b) A', (c) C" and (d) A", and for (iv) using Methods (e) C and (f) A' (Methods C" and A"' are omitted), whereas Figs. 5a and 5b respectively show those obtained for (v) and (vi) using Method A"'. For (iii) and (iv) with Method $\mathrm{C}$, the mechanical source reconstructions obtained for $0.25 \mathrm{sec}$ are also shown (i.e., Fig. $4 \mathrm{a}$ and $4 \mathrm{e})$.

Except for the sinusoidal [(iii)] and step [(iv)] pressures using Method C, accurate shear modulus 
(a)
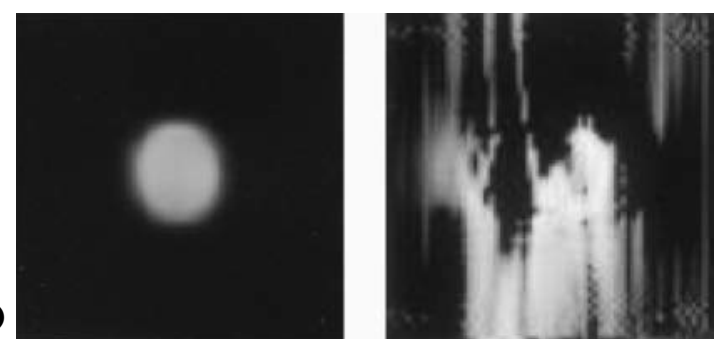

(b)

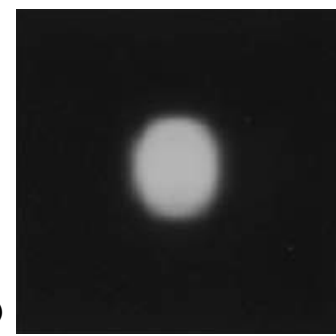

(c)

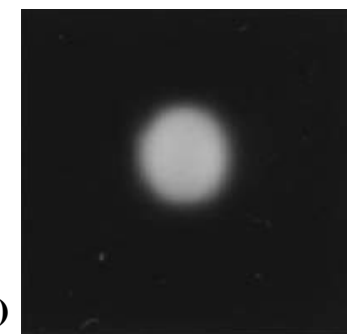

(d)

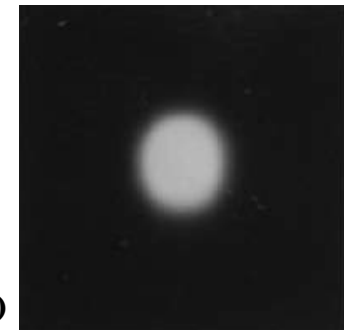

(e)
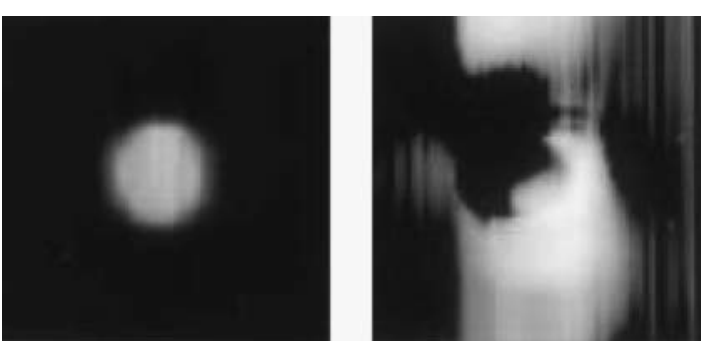

(f)

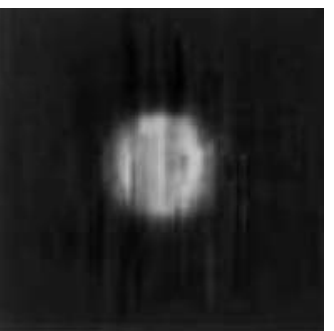

2.03

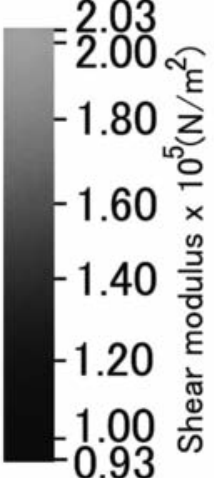

Fig. 4. Shear modulus reconstructions obtained for internal mechanical sources (vertical central plane, $y=25$ $\mathrm{mm}$ ) : (iii) sinusoidal pressure p' using Methods (a) C, (b) A', (c) C", and (d) A"' ; (iv) step pressure p' using Methods (e) C and (f) A'. For (a) and (e) using Method $\mathrm{C}$ (i.e., six unknowns), inaccurate and unstable mechanical source reconstructions are also shown (right), of which dynamic ranges are determined by the respective ranges of the reconstructions (respective means, 2.69 and $5.28 \times$ $\left.10^{-7} \mathrm{~N} / \mathrm{m}^{2}\right)$. 
(a)
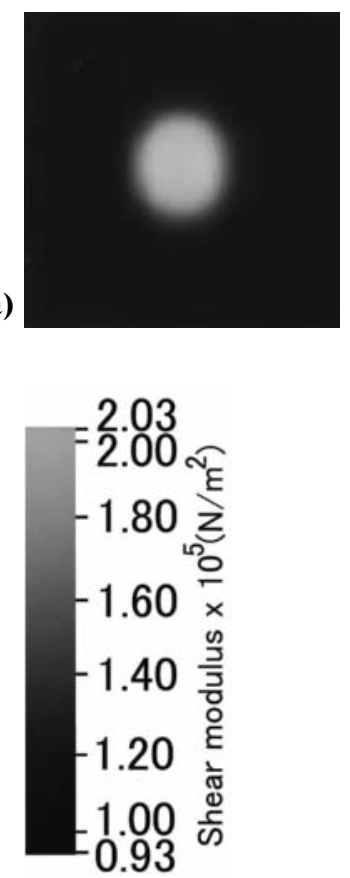

(b)

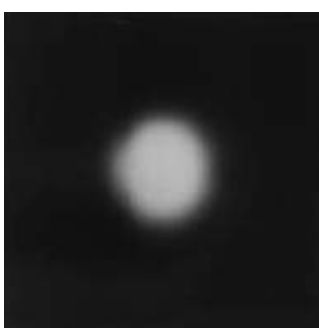

Fig. 5. Shear modulus reconstructions obtained for an internal mechanical source (vertical central plane, $y=25 \mathrm{~mm}$ ): (a) sinusoidal 3D force vector hi' $[(\mathrm{v})]$ and (b) a step 3D force vector hi' [(vi)] using Method A"”.

reconstructions were obtained with Method C and combined reconstructions with Methods A', C" and A" (see Table IV, 1.81 and 1.82 vs 1.89 to $2.06 \times 10^{5} \mathrm{~N} / \mathrm{m}^{2}$ ). When using a step pressure or force with Method C (i.e., six unknowns), the shear modulus reconstruction values decreased. In addition, for the mechanical source reconstructions theoretically achievable by Method $\mathrm{C}$, inaccurate and unstable reconstructions were obtained. See the very small means obtained $\left(e . g\right.$., order of $\left.10^{-7} \mathrm{~N} / \mathrm{m}^{2}\right)$. Thus, the large number of unknowns degrades the mechanical source reconstructions particularly.

\section{Conclusions}

Through 3D simulations, for static and dynamic mechanical sources, it was possible to reconstruct the shear modulus when internal mechanical sources exist. In the simulations, an external mechanical source was also used simultaneously. This was also possible in such reconstructions, when combining an internal mechanical source with other terms except for the shear modulus, e.g. inertial and mean normal stresses. For the purpose of shear modulus reconstruction, such a decrease in the number of unknowns decrease the computational time and increase the reconstruction accuracy and stability. Recall that the large number of unknowns such as six unknowns yielded inaccurate and unstable dynamic mechanical source reconstructions even though any measurement noise is not dealt with. For the next trials, the regularization ${ }^{3-5}$ and the regulation ${ }^{4,5}$ previously developed for a shear modulus reconstruction with other unknowns will be used for coping with interfering noise together with the decrease in the accuracy and stability of the mechanical source reconstructions.

However, for the preliminary trials, the performed simulations show that the extended reconstruction methods have a high potential to produce an arbitrary internal mechanical source reconstruction such as a HIFU, static compressors, vibrators, heart motion and pulsation together with the shear modulus 
reconstruction.

Thus, as mentioned earlier, the developed mechanical source reconstruction methods will increase the applications for shear modulus reconstruction, e.g. for dynamic or static cells and tissues such as cardiac cells, blood vessels, skin, muscle, cells and tissues in culture, for imaging and monitoring during treatments and diagnoses respective with a HIFU and radiation force transmission, for the estimation of a PSF for designing an US beamformer for US imaging, for controlling the radiation force for imaging tissue deformation or shear wave propagation (i.e. elasticity imaging), and for HIFU treatments. However, also note that when the HIFU or radiation force transmission is performed, shear modulus reconstructions can also be performed using the previous Methods A to F by dealing with the cessation of the internal mechanical sources similarly to the thermal inverse problem that deals with the cessation of heating and perfusion ${ }^{17)}$. If a mechanical source can be estimated in advance analytically, numerically, or experimentally, the shear modulus reconstruction can also be performed using the same equations and the mechanical source data. Moreover, for these applications, after the shear modulus reconstructions using external sources ${ }^{3-6)}$, internal sources can also be reconstructed using the measured strain tensor data. This reconstruction also corresponds to the simultaneous reconstructions of internal mechanical sources and mechanical properties using the prime numbers of deformation fields shown in Tables II and III (simulations not shown). That is, the number of unknown variables decreases. Especially, for the purpose of internal mechanical source reconstructions, for Methods A, and D and E using $\mathrm{A}$ in a dynamic deformation case, the combination of an inertia and a mean normal stress of the same deformation field dealt with as an unknown permits no combined internal mechanical source reconstructions similarly to Methods F, A to C, and D and E using Methods F and A to C in the static deformation case (Table II) and in the dynamic deformation case (Table III). The methods are referred to as Methods A"', and D"' and E"' using A"” as shown in Table V in appendix. In conjunction, when internal sources do not exist, the combination of an inertia and a mean normal stress is also effective for a shear modulus reconstruction similarly. These reconstructions will be reported elsewhere.

Here, we also need to mention that in $1 \mathrm{D}$ and 2D reconstructions, the use of a small mechanical source yields shear moduli which are smaller than the original (data omitted). This is because, in such low dimension reconstructions, both internal and external mechanical sources affect the shear modulus and source reconstructions as occurs with solo shear modulus reconstructions ${ }^{3-5)}$ owing to the low number of dimensions, so it is necessary to evaluate the effects of mechanical source conditions (e.g. size, intensity, and frequency) on such low dimension reconstructions. This is also a future problem.

The case where the reconstruction target is a stress tensor ${ }^{8)}$ should also be mentioned. As described above, a mean normal stress can be reconstructed ${ }^{4,5)}$. Hydrostatic pressure in fluid can also be reconstructed together with fluid parameters in the same manner. Thus, other stress tensor components can be evaluated using the reconstructed mechanical properties and mean normal stress or hydrostatic pressures together with measured deformations. In a manner similar to shear modulus reconstructions, viscous shear modulus reconstructions can also be carried out by measuring strain tensor rates together with strain tensors and acceleration vectors ${ }^{18)}$. 
Table V. For the purpose of no combined internal mechanical source, in a dynamic deformation case, the number of independent fields required by Methods A"', and D"," and E"," using A"', with the number of unknowns. The prime numbers show the numbers of deformation fields required for reconstructing the internal mechanical sources that exist only in one deformation field using another deformation field together.

\begin{tabular}{|c|c|c|}
\hline Method (and ref.) & \multicolumn{2}{|c|}{$A ", "(G), D "$, and E", $(\bar{G})$ using A"," } \\
\hline Number & Fields & Unknown \\
\hline Pressure p' & 1 & $3(\mathrm{G}, \mathrm{p}, \mathrm{comb})$ \\
\hline $\begin{array}{l}\text { 3D force } \\
\text { hi' }(i=1-3)\end{array}$ & $2^{\prime}$ & $6(\mathrm{G}, \mathrm{hi}, \mathrm{comb} \times 2)$ \\
\hline $\begin{array}{l}\text { 2D force } \\
\text { hi' }(i=1,2)\end{array}$ & $2^{\prime}$ & $5(\mathrm{G}, \mathrm{hi}, \mathrm{comb} \times 2)$ \\
\hline A force $h^{\prime}$ & 1 & $3(\mathrm{G}, \mathrm{h}, \mathrm{comb})$ \\
\hline
\end{tabular}

In the table, $\mathrm{G}$ is the shear modulus, and comb is obtained by combining an inertia $\rho(\rho \alpha)$ and a mean normal stress $\mathrm{p}$ in Methods A, and D and E using A. When using Methods D"', and E"', quasi-reconstructions of mechanical sources are obtained (not shown in table).

\section{Appendix. Shear modulus reconstructions especially for the purpose of no combined internal mechanical source reconstructions}

The simultaneous reconstructions of internal mechanical sources and mechanical properties using the prime numbers of deformation fields are shown in Tables II and III. The prime numbers show the numbers required for reconstructing the internal mechanical sources that exist only in one deformation field using another deformation field together. Especially, for the purpose of no combined internal mechanical source reconstructions under the same conditions, for Methods A, and D and E using A in a dynamic deformation case, the combination of an inertia and a mean normal stress of the same deformation field dealt with as an unknown permits no combined mechanical source reconstructions similarly to Methods F, A to C, and D and E using Methods F and A to C for the static deformation case (Table II) and for the dynamic deformation case (Table III). The methods are referred to as Methods A"", and D"" and E"' using A"" as shown in Table V. These reconstructions correspond to those performed after the shear modulus reconstructions using external sources ${ }^{3-6)}$.

\section{References}

1) Sumi C., Matsuzawa H. : Shear modulus reconstruction by ultrasonically measured strain ratio. J Med Ultrasonics, 34 : 171-188, 2007.

2) Sumi C. : Comparison of contrast-to-noise ratios of axial strain, shear modulus and inverse of shear modulus estimated by axial strain ratio. IEEE Trans Ultrasonics Ferroelectrics Frequency Control, 56: 669-675, 2009.

3) Sumi C.: Usefulness of ultrasonic strain measurement-based shear modulus reconstruction for diagnosis and thermal treatment. IEEE Trans Ultrasonics Ferroelectrics Frequency Control, 52: 1670-1689, 2005.

4) Sumi C.: Reconstructions of shear modulus, Poisson's ratio and density using approximate mean normal stress $\lambda \varepsilon_{\alpha \alpha}$ 
as unknown. IEEE Trans Ultrasonics Ferroelectrics Frequency Control, 53 : 2416-2434, 2006.

5) Sumi C. : Effective shear modulus reconstruction obtained with approximate mean normal stress remaining unknown. IEEE Trans Ultrasonics Ferroelectrics Frequency Control, 54 : 2394-2403, 2007.

6) Sumi C.: Useful relative shear modulus reconstruction for visualization with no geometrical artifact. Acous Sci Techno, in press.

7) Sumi C., Tanuma A.: Comparison of parabolic and Gaussian lateral cosine modulations in ultrasound imaging, displacement vector measurement, and elasticity measurement. Jpn J Appl Phys, 47 (5B) : 4137-4144, 2008.

8) Sumi C. : Beamforming for realizing designed point spread function. "Proceedings of IEEE Int Ultrason Symp". pp. 1557-1562, 2007.

9) Sumi C.: Increasing accuracy of tissue shear modulus reconstruction using ultrasonic strain tensor measurement Regularization and lateral modulation. Acoustical Imaging, 29: 59-69, 2008.

10) Muthupillai R., Lomas D.J., Rossman P.J., Greenleaf J.F., Manduca A., Ehman R.L.: Magnetic resonance elastography by direct visualization of propagation acoustic strain waves. Science, 269: 1854-1857, 1995.

11) Sugimoto T., Ueda S., Itoh K. : Tissue hardness measurement using ultrasonic radiation force -theory and experiment using Voigt model. Jpn J Medical Ultrasonics, 20 : 277-283, 1993 (in Japanese).

12) Bercoff J., Tanter M., Fink M. : Supersonic shear imaging: a new technique for soft tissue elasticity mapping. IEEE Trans Ultrasonics Ferroelectrics Frequency Control, 51 : 396-409, 2004.

13) Dahl J.J., Pinton G.F., Palmeri M.L., Agrawal V., Nightingale K.R., Trahey G.E.: A parallel tracking method for acoustic radiation force impulse imaging. IEEE Trans Ultrasonics Ferroelectrics Frequency Control, 54 : 301-312, 2007.

14) Sumi C., Kanada H., Takanashi Y., Shimizu K., Sato N., Uchida T., Minami A.: Thermal property reconstruction-Reconstruction of thermal source and perfusion. "Proceedings of $7^{\text {th }}$ Int. Conf. Ultrason. Meas. Imag. Tissue Elasticity", 2008.

15) Sumi C., Yanagimura H.: Reconstruction of thermal property distributions of tissue phantoms from temperature measurements -Thermal conductivity, thermal capacity and thermal diffusivity. Phys Med Biol, 52: 2845-2864, 2007.

16) Sumi C., Yanagimura H. : Spatial inhomogeneity of tissue thermal parameter of Ebbini's model and its dependency on temperature. Jpn J Appl Phys, 46: 4790-4792, 2007.

17) Sumi C., Uchida T., Minami A.: Thermal property reconstruction in tissues using temperature distribution data obtained after the cessation of heating and perfusion. Thermal Med, 24 : 91-99, 2008.

18) Sumi C.: Usefulness of ultrasonic strain measurement-based mechanical properties imaging technique: toward realization of short-time diagnosis/treatment. "Research and Development in Breast Ultrasound", Eds. E. Ueno, T. Shiina, M. Kubota, K. Sawai, Springer-Verlag, pp.16-43, 2005. 